\title{
A Developmental Model of Infantile Nystagmus
}

\section{Prof. Chris Harris \\ SensoriMotor Laboratory, \\ Centre for Theoretical and \\ Computational Neuroscience, University of Plymouth, Plymouth, Devon, UK \\ David Berry \\ Departmento de Fisica e Centro de Geofisica de Evora, Universidade de Evora, Colegio Luis Verney Evora, Portugal}

\begin{abstract}
The possibility that infantile nystagmus (IN) may reflect a failure in early sensorimotor integration has been proposed for more than a century, but is only recently being borne out in animal studies. The underlying neural and genetic substrate for this plasticity is complex. We propose that, in most cases, IN develops as a developmental response to reduced contrast sensitivity to high-spatial frequencies in an early "critical period," however caused, whether by structural malformations (e.g. foveal hypoplasia) or poor optics (e.g. cataract). As shown by psychophysics, contrast sensitivity to low spatial frequencies is enhanced by motion of the image across the retina. Based on our previous theoretical study (Harris \& Berry, Nonlinear Dynamics, 2006), we argue that the best compromise between moving the image and maintaining the image near the fovea (or its remnant) is to oscillate the eyes with jerk nystagmus with increasing velocity waveforms, as seen empirically. The generation of jerk waveforms relies heavily on the saccadic system, which is immature in infancy. Pendular waveforms may therefore provide an alternative to jerk waveforms, and may explain why they are seen more often in young infants. We discuss the implications of this developmental model for the need to synchronize sensory and motor developments in normal development. Failure of this synchronization may also explain some idiopathic cases.
\end{abstract}

KEYWORDS congenital nystagmus, infant eye movements, oscular oscillations, developmental plasticity, visual development, optimal control

In the congenital cases [of nystagmus] it is probable that the absence of the stimulus that accurate retinal impressions afford interferes with the functional development of the co-ordinating centers for the orbital muscles [Swanzy, 1895, p542].

\section{INTRODUCTION}

We would like to thank Faith Budge and Noreen Derbyshire for their help in preparing this study, and Cerebra for their financial support.

Address correspondence to Chris Harris, SensoriMotor Laboratory, Centre for Theoretical and Computational Neuroscience, University of Plymouth, Plymouth, Devon PL4 8AA, UK. E-mail: cmharris@plymouth.ac.uk
Infantile nystagmus (IN) is a great challenge for developmental neuroscience, as well as a continuing frustration for affected families and clinicians. IN has been extensively studied phenomenologically, and has attracted systems modellers for 30 years or more. Yet IN remains largely intractable and we seem no closer to understanding why some infants exhibit wobbly eyes and others do not. In this paper we outline a new modelling approach which focuses on IN as a developmental process in which abnormal post-natal visual experience induces an adaptive oculomotor response that leads to nystagmus during a "critical" 
period of heightened plasticity. This approach is based on our recent mathematical study (Harris \& Berry, 2006).

The onset of IN is before 6 months of age. Unlike acquired (neurological) nystagmus, no consistent brain lesion has been identified. Instead, the majority of affected patients have one of a very wide range of visual abnormalities (such as albinism, achromatopsia, aniridia, cataract, coloboma, corneal opacities, and many more). Thus it is implausible to attribute the cause of IN to any specific visual pathway abnormality, or even to a single gene. The only common feature among the sensory deficits is that they are present around the time of birth and usually affect both eyes. Visual deficits with an onset later in infancy (such as cataracts) do not lead to IN. Thus, there is a strong case that IN is an anomaly of early sensorimotor development. The first few months of life are a period of rapid oculomotor development. We propose that this motor development is plastic and can be influenced by post-natal visual experience. Indeed, it is well established that early monocular visual deprivation can lead to a constellation of abnormal oculomotor development (latent nystagmus, strabismus, OKN abnormalities). In a similar vein, IN may result from early binocular deprivation (Tusa et al., 2001). It also seems that this plasticity may be under active genetic control, which can itself be influenced by visual experience (McCullen et al., 2004). The key issue, therefore, is to achieve a better understanding of early visuomotor plasticity.

We propose that there is a developmental "funnel" in which any antecedent sensory abnormality will divert oculomotor plasticity towards IN. We propose that this funnel is a loss of high spatial frequency information relative to the normal infant, however caused-whether by structural retinal abnormalities (e.g. foveal hypoplasia) or visual deprivation (e.g. poor optics as in cataracts). Our premise is that the 'goal' of early oculomotor plasticity is to develop a control system that maximizes visual contrast. For the normal foveate (or "foveate-to-be") retina, this leads to a strategy of holding the image of an object on the fovea, which we call "fixation" and "smooth pursuit" for stationary and moving targets, respectively. However, we hypothesize that this is not inevitable, and when high-spatial frequency contrast is not available, plasticity could lead to oscillatory (nystagmoid) strategies.

\section{PLASTICITY AND OPTIMIZATION}

Plasticity is the ability of a phenotype to modify its behavior and/or structure based on experience of the external environment. Obviously plasticity occurs at different levels, such as cognitive learning, low-level adaptive control, and structural development. Implicit is the notion that this self-modification is driven by the interaction between the phenotype and its environment, which in the normal individual leads to adaptive behavior. We can consider plasticity in fundamentally different ways. We can take a "proximal" approach by finding how plasticity is mediated (i.e. the underlying genetic, structural and neural substrates), or we can take a "distal" approach by trying to understand the goal or objective of the plasticity. We consider the latter.

If we can state the goal of plasticity we can, in principle, find the ultimate behavior of such a plastic process without knowing the neural, structural or genetic mechanisms. To do this, we couch the adaptive process as an optimization problem where a cost (benefit) function is minimized (maximized) to yield the final state of the plastic process, subject to physical and physiological constraints. This approach makes no assumptions about how the goal is achieved and is applicable with or without error feedback control (Harris, 1998).

We shall assume that the goal of early oculomotor plasticity is to develop an oculomotor system that controls the trajectory (dynamical state: position, velocity, etc.) of the image of an object to maximize visual contrast, $C$. Because of the spatial and temporal response of the visual system, contrast will depend on the shape and size of the object's retinal image and on the motion of the image across the retina, which we call the image state vector $\mathrm{x}(t)$ (position, velocity, acceleration, etc.), so that contrast obviously depends on the image state $C=C(\mathrm{x})$. The final outcome of plasticity will be to attain state trajectories that reach a local maximum of $C(\mathrm{x})$ for a given set of constraints, and to develop a control system that maintains these trajectories. How this is achieved remains unknown. One could view the initial system as a highly flexible adaptive system in which the parameter space can be reduced around potentially different attractors. The final attractor is then the "normal" behavior, which is maintained by the same adaptive system with its reduced adaptive range. Alternatively, one could view the developing system as a meta-control system that has the potential to develop many different adaptive control systems. However, this 
hierarchical approach is really only a metaphor since ultimately all levels of control are realized by connectivity at the neural level. Any making or pruning of connectivity in the neonatal brain will affect all levels.

Because of the influential control systems history in the study of oculomotor control, it must be emphasized that this process is not necessarily an error-feedback system where retinal slip is minimized to yield fixation and smooth pursuit. Such a system would only be one of many systems that could develop.

\section{THE SPATIOTEMPORAL CONTRAST RESPONSE SURFACE}

From psychophysics, it is well known that contrast sensitivity to a visual grating is optimized by some degree of stimulus motion, where the optimal speed is an inverse function of grating spatial frequency. For the highest spatial frequencies the optimal speed is very small (although not zero) (Kelly, 1985), but at low spatial frequencies the optimal speed reaches tens of degrees per second. This velocity tuning can be predicted from the low-level spatial and temporal tuning of center-surround receptive fields (RFs) of retinal ganglion cells, since velocity can be considered as temporal frequency divided by spatial frequency (Kelly, 1985). The optimal speed for a grating of a given spatial frequency also increases with foveal eccentricity (Virsu et al., 1982), which is consistent with the increasing size of RFs (or the loss of high spatial frequency sensitivity) with eccentricity. Velocity tuning also occurs with simple visual targets such as bars. For example, the optimal speed of a bar with a width of 1 degree is about $2 \mathrm{deg} / \mathrm{s}$, but this increases to $10 \mathrm{deg} / \mathrm{s}$ for a bar with a width of 3 degrees (Burr \& Ross, 1982). Optimal speed also increases with eccentricity for fine lines as for gratings (Kelly, 1985). We argue that if the visual system does not have high spatial frequency sensitivity due to the absence or lack of stimulation of foveal RFs, overall contrast sensitivity will be enhanced by some degree of image motion. However, image motion would move the image off the central retina onto the peripheral retina where contrast sensitivity would again be reduced. Thus, the question is what kind of image motion would maximize contrast?

To capture this spatiotemporal sensitivity we consider contrast to be a function of image position relative to the fovea, $y(t)$, and as a function of speed of the image moving across the retina, $\dot{y}(t) \equiv \frac{d y}{d t}$; that is $C=C(y, \dot{y})$. It is important to recognize that this function is not simply binary, with contrast sensitivity being maximal for speeds below some proscribed speed and absent above that speed, as used in the definition of a "foveation period" (DellOsso et al., 1992). $C(y, \dot{y})$ is a 2-D function extending smoothly to high eccentricities and to high speeds, and is actually a contrast response function for supra-threshold stimuli. We denote the optimal speed (for a given stimulus) by $p(y)$.

For a stimulus with high spatial frequencies, $C(y, \dot{y})$ will have a maximum very near the phase space origin $[y=0, \dot{y}=0$, or $(0,0)]$ for a normal functioning foveate retina, because $p(0) \sim 0$. For a retina without sensitivity to high spatial frequencies (or a stimulus without high spatial frequencies), $p(0)>0$ and the maximum of $C(y, \dot{y})$ will be at $(0, p)$. Motion that maintains constant optimal position and constant optimal non-zero speed is not possible except in the limiting case of a sawtooth trajectory with infinitessimal amplitude and infinite frequency. However, the visual system would be insensitive to contrast at such high temporal frequencies, so the optimum will not be at $(0, p)$, and clearly we need to consider contrast as a dynamic function.

To find an approximate optimal solution without modelling the whole visual system, we consider the lack of sensitivity to high temporal frequencies as equivalent to a visual integration time, $T$, and write contrast as an integral:

$$
C=\int_{0}^{T} F(y, \dot{y}, t) d t
$$

where $F($.) is some well-behaved physiological function. Following the traditional mathematical approach to solving this type of problems, we assume that $\mathrm{F}($.$) is$ smooth around $(0, p)$ so that it can be described by a parabola close to $(0, p)$. In the region of $(0, p)$, contrast is then given by:

$$
C=C_{\max }-\int_{0}^{T}\left[a(y(t))^{2}+b\left(\dot{y}(t)-p(y)^{2}\right)\right] d t .
$$

Here $C_{\max }$ is the maximum contrast at $(0, p)$, and $a$ and $b$ are constants that reflect the difference in contrast loss when the image moves away from the fovea or away from the optimal speed, $p$. Simplifying further, we assume that there is negligible change in $p(y)$ around $(0, p)$ so that $p=p(0)$. It can then be shown (Harris \& Berry, 2006) that the optimal trajectory is given by the 
sum of two exponents:

$$
y(t)=A e^{-t / \tau}+B e^{t / \tau},
$$

where $\tau=\sqrt{b / a}$. The constants $A$ and $B$ are determined by boundary conditions (if any) and will depend on $p$. In the normal visual system, $p \sim 0$, the optimal strategy is $y(t) \sim 0$, i.e. to keep the image on the fovea. It is important to recognize that $y(t)$ is image position (not eye position). If the object and head are stationary then the optimal image motion is generated by the eye movement $e(t)=-y(t)$. If the object is moving, $o(t)$, then the optimal image motion is $y(t)=-o(t)-e(t)$, and it will be necessary to generate smooth pursuit with optimal eye movement superimposed. We will not pursue this here and assume a stationary object and head.

\section{JERK NYSTAGMUS}

When the optimal image speed, $p$, differs substantially from zero, the optimal strategy is to move the image as a compromise between matching $p$ and keeping its position close to the region of highest spatial resolution at $\mathrm{y}=0$ (which for the sake of simplicity we will continue to call the "fovea"). In general, the ideal image movement will cause a net change in position image, so that for extended temporal processing of the image, image position will need resetting, ideally instantaneously. For the oculomotor system, the fastest resets available are saccades (which are not instantaneous), leading to jerk nystagmus as the near-optimal strategy. The timing and metrics of saccadic quick phases (QP) then become a crucial issue as they place limits on the resetting.

Little is known about QPs, other than they are saccade-like in their trajectories (Harwood, 2003). As a rule patients with jerk IN are not aware of their quickphases (but are aware of their voluntary saccades). In this respect, IN QPs are similar to optokinetic QPs. One peculiarity of IN QPs is that they can reset ongoing slow eye movement velocity. Thus, eye velocity at the end of a slow phase may be very high but may be reduced to near zero after the QP. We have assumed that this is a feature of the gaze-holding apparatus rather than the QP per se (Harris, 1995). It is not clear what triggers IN QPs. OKN QPs have an increased frequency and amplitude when the stimulus velocity increases, but there is considerable variability in timing and amplitude from one cycle to the next. In infants, nystagmus frequency is typically much lower than in adults, and the nystagmus has a much larger and more variable amplitude

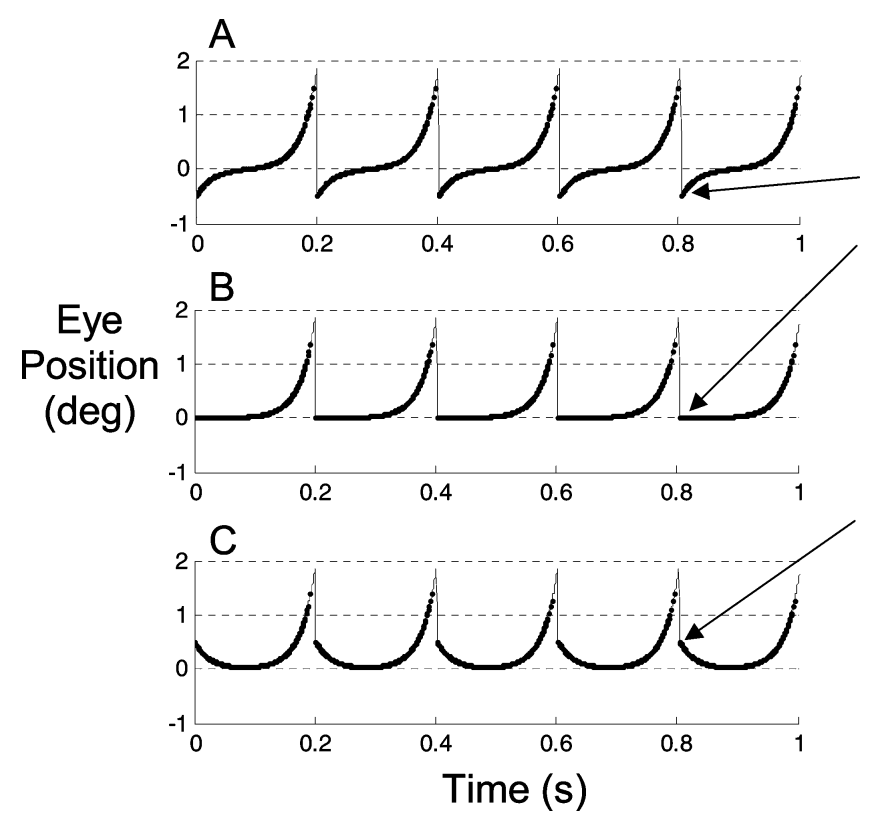

FIGURE 1 Theoretically optimal trajectories for different quickphase end positions (solid arrow) a) hypermetric quick-phase bringing the fovea back beyond the target by $0.5 \mathrm{deg}$ (taking the image across the fovea); b) Foveating quick-phase; c) hypometric quick-phase with fovea falling short by $0.5 \mathrm{deg}$ (pseucocycloid). Parameters: $T=0.15 \mathrm{~s}, \mathrm{p}=10 \mathrm{deg} / \mathrm{s}, \mathrm{a}=\mathbf{0 . 0 2 5}$. Slow phase duration was set to $D=0.2 \mathrm{~s}$.

(Harris et al., 1994; Garbutt et al., 2006). It is unlikely, therefore, that QP production can be controlled sufficiently to generate the ideal image motion, especially in infancy, and we need to consider the effects of inaccuracy and variable timing.

The effect of a QP inaccuracy is to start the next nystagmus slow phase at a different starting position, $y(0)$. We can calculate the optimal trajectory for a given $y(0)$ and the general result is shown in Figure 1 (Harris \& Berry, 2006). For QPs that overshoot (i.e. that bring the image back past the fovea), the optimal trajectory is a mixed decelerating and accelerating slow phase (Figure 1a); for a precisely foveating QP a purely accelerating slow phase is optimal (Figure 1b); and for an undershooting QP the optimal trajectory is a pseudocycloid waveform (Figure 1c). All of these trajectories are observed in IN, although, to our knowledge, their relationship to retinal landmarks has not been empirically examined.

The effect of timing variability is more complex. If the QP is not generated until some time after $T$, what should the optimal image motion be? Let us assume that the average slow-phase duration is $\bar{D}$, where $\bar{D}>T$. One possibility is that the cost is minimized over $\bar{D}$ rather than $T$. This would yield the same trajectory shapes as shown in Figure 1 but spread over $\bar{D}$ rather 
than $T$. Thus, the maximum velocity over $\bar{D}$ would still be $p$. Then individual slow phases that were briefer than $\bar{D}$ would be foreshortened, and those longer than $\bar{D}$ would be extended in time. We would expect extended slow phases to reach very high velocities because of the high accelerations. An alternative, and perhaps counterintuitive, strategy would be to accelerate the image motion as much as possible after $T$ has elapsed. If QPs are more likely to be triggered at higher speeds, then rapid acceleration would induce a QP more quickly and with less timing variability.

The key point is that in an adaptive process (as we are assuming here) QPs would affect the slow phase just as slow phases affects the QPs, resulting in a complicated cyclical process and, in our opinion, justifying nonlinear dynamical systems analysis (Abadi et al., 1997; Harris \& Berry, 2006). Experimentally, understanding the mechanisms of QP production becomes essential in order to find how the optimal waveform is constrained by QP timing.

\section{PENDULAR NYSTAGMUS}

Although QPs are the fastest resetting mechanism available to the oculomotor system, they are clearly not without problems. Accuracy and timing issues may lead ultimately to a rather poor control of image motion, in which waveforms may be considerably suboptimal. In infancy, when IN develops, saccade production is particularly variable, and an alternative strategy would be to oscillate the image position without saccadic eye movements, in quasi-sinusoidal fashion. In other words, reset position slowly if it can be done accurately. IN in infancy is often pendular before developing into jerk (Reinecke et al., 1988).

It has been shown that the normal smooth pursuit is capable of oscillating quasi-sinusoidally (Robinson et al., 1986) and this property has already been suggested as a possible source of IN (Harris, 1995; Jacobs $\&$ Dell'Osso, 2004). Consider the image motion to be a simple deterministic sinusoid with amplitude $A$ degrees and frequency $f$ :

$$
y(t)=A \cos (2 \pi f t)
$$

If we assume an isotropic retina, where the optimal speed $p$ may be in either direction (on each half cycle), we can evaluate cost according to Eq. 2 over a half cycle, and it can be shown that the amplitude that minimizes cost for a given frequency of oscillation is approximately inversely proportional to the frequency:

$$
A \approx \pm 2 p / \pi^{2} f
$$

A high frequency would require a low amplitude of oscillation. This makes sense since increasing amplitude or frequency will increase peak velocity of the motion. It is in qualitative agreement with the observation that a high frequency IN usually (if not always) has a very low amplitude. One could consider a Fourier sum of sinusoids to yield more complex shapes, as seen empirically in the asymmetric pendular waveform (Dell'Osso \& Daroff, 1975), but again we need to know more about the limitations or costs in generating such waveforms. We have also assumed that there is no noise in the oscillation. There will inevitably be fluctuations in any biological oscillating system, which will complicate the problem further. Although mathematically simple, we should also recognize that a steady sinusoid is not a simple mode of oscillation, but should be considered as a limit cycle in a non-linear system.

\section{DISCUSSION}

The idea that IN may result from a failure of sensorimotor integration in infancy is not new, and can be traced back over a century (Swanzy, 1895). More recently it has been shown that visual deprivation can lead to IN-like oscillations in monkey (Tusa et al., 2001). The plausibility of this is further ramified by the demonstration that cascades of gene expressions can be affected by early visual deprivation in the (afoveate) mouse (McCullen et al., 2004), which not only blurs the naturenurture division, but provides a rather daunting preview into the complexity of genetic control of normal and abnormal visuomotor development. The companion of complexity is vulnerability to error, and the question arises how control is maintained in spite of genetic polymorphisms. We have attempted to side-step these fundamental proximal issues by assuming that plasticity is indeed under control, with some kind of evolutionary "goal". By modelling the goal as maximizing visual contrast when high spatial frequency information is not available, we can reproduce many of the oscillatory jerk waveforms peculiar to IN.

Another key concept that emerges when we consider optimal strategies is how closely can the oculomotor system approach the theoretical ideal. What are the constraints imposed by the infant oculomotor system? For 
example, timing of resetting quick-phases is highly variable in infants (at least for OKN), with slow phases duration being much longer than in adults. Thus, it seems reasonable to argue that the infant oculomotor system would not be able to generate the ideal waveform, and slow phases would become extended in time. This would increase the cost of jerk waveforms considerably. Pendular waveforms do not need saccades and their frequency and amplitude tend to be relatively stable (at least when compared to jerk waveforms). Thus, pendular waveforms may be an acceptable alternative waveform. The interplay between slow and quick phases becomes an important issue, but we need more experimental data to take this further.

It is possible, therefore, that for the young infant the adaptive process generates quasi-sinusoidal waveforms due to the large cost of poor fast phase timing. As the saccadic system matures in the older infant, jerk waveforms would become preferable. Indeed it seems trivially easy to make this transition since a resetting fast phase would stop a sinusoidal cycle and convert it to an accelerating slow phase. Although it is possible to observe examples that are supportive of this idea, some caution is needed. First, pendular oscillations can be of very high frequency and low amplitude even in the young infant. Simply interrupting each cycle with a quick phase seems unlikely, and in any case would not yield the typically slower jerk nystagmus. Second, the fast phase would need to reset velocity, otherwise there would be no advantage. It should be noted that QPs in physiological nystagmus (optokinetic or vestibular) do not reset eye velocity, so some special mechanism needs invoking.

Jerk nystagmus tends to be strongly gaze-dependent, implying involvement of the gaze-holding circuitry. It seems possible to approximate the ideal jerk waveforms by the gaze-holding circuitry alone, which we are currently exploring. This would inevitably lead to a null region (Harris \& Berry, 2006), which is consistent with observation. However, high frequency pendular nystagmus may be independent of gaze, implying a different pre-motor source. One possibility is the smooth pursuit system, as recognized previously (Harris, 1995; Jacobs \& Dell'Osso, 2004), although we are arguing that this would be an adaptive strategy rather than a clinical accident.

If lack of sensitivity to high spatial frequencies in early infancy is a sufficient condition for IN to develop, should not the normal neonatal macular immaturity cause all infants to develop IN? This is one of the major questions for this model and any other sensory-defect argument. To address this we need to consider the timeline of development. Although image motion (with the ideal waveform) would improve visual contrast in early infancy, it would not be beneficial when the fovea matures. On the other hand, high gain smooth pursuit and fixation would maximize visual contrast for the fovea when it does develop. Evolution would need to tread a fine line by programming the development of oculomotor control in tandem with foveal maturation to maximize visual contrast without causing nystagmus. Modulating plasticity could prevent oculomotor development from outpacing sensory development. This seems a plausible explanation for the postnatal development of smooth pursuit in the first place. Failure of this synchonization through delayed sensory development in the presence of normal motor development, or precocious oculomotor development in the presence of normal sensory development, would then lead to IN. Recent studies in mice have shown that early visual deprivation can lead to a whole cascade of changes in gene expressions for extraocular muscle, oculomotor systems (delayed OKN \& VOR) and neural plasticity (McCullen et al., 2004). It is likely that there is a similar genetic control of foveal development and plasticity in the eye movement systems that support foveal function (gaze holding, smooth pursuit, fast OKN). Indeed, these oculomotor systems can be switched off in the second year of human life due to a mutation on chromosome 13 (Ragge et al., 2003).

It is tempting to speculate that disruption in the timing of this developmental sequence through delayed sensory or precocious oculomotor development might explain idiopathic IN, including "idiopathic" nystagmus in Down's syndrome (Lawson et al., 1996), and transient idiopathic nystagmus in infancy (Good et al., 2003).

\section{REFERENCES}

Abadi RV, Broomhead DS, Clement RA, Whittle JP, Worfolk R. Dynamical systems analysis: a new method of analysing congenital nystagmus waveforms. Exp Brain Res. 1997; 117:355-61.

Burr DC, Ross J. Contrast sensitivity at high velocities. Vision Research $1982 ; 22: 479-84$

Dell'Osso LF, Daroff RB. Congenital nystagmus waveforms and foveation strategy. Documenta Ophthalmologica 1975; 39:155-82.

Dell'Osso LF, Van Der Steen J, Steinman RM, Collewijn H. Foveation dynamics in congenital nystagmus. I: Fixation. Documenta Ophthalmologica 1992; 79:1-23.

Garbutt S, Harwood M, Harris CM. Infant saccades are not slow. Developmental Medicine \& Child Neurology 2006; (in press). 
Good WV, Hou C, Carden SM. Transient, idiopathic nystagmus in infants. Developmental Medicine \& Child Neurology 2003; 45:304-7.

Harris CM, Jacobs M, Taylor D. The development of bi-ocular and monocular optokinetic gain from 1 to 7 months. Investigative Ophthalmology and Visual Science (Suppl) 1994; 1829.

Harris CM. Problems in modelling congenital nystagmus: towards a new model. In Eye Movement Research: Mechanisms, Processes and Applications 1995; Findlay JM, Walker R, Kentridge RW (eds). Elsevier: Amsterdam, 239-53.

Harris CM. On the optimal control of behaviour: a stochastic perspective. Journal of Neuroscience Methods 1998; 83:73-88.

Harris CM, Berry D. A distal model of congenital nystagmus as non-linear adaptive oscillations. Nonlinear Dynamics 2006; (in press).

Harwood M. Fourier analysis of saccades. PhD Thesis, University College, London (2003).

Jacobs JB, Dell'Osso LF. Congenital nystagmus: hypotheses for its genesis and complex waveforms within a behavioural ocular motor system. Journal of Vision 2004; 4:604-25.

Kelly DH. Visual processing of moving stimuli. J Opt Soc Am A 1985; 2:215-25.

Lawson J, Shawkat F, Timms C, Thompson D, Kriss A, Russell-Eggitt I, Taylor D, Harris CM. Eye movements in children with Down's syn- drome. Investigative Ophthalmology and Visual Science (Suppl) 1996; 37:278.

McCullen CA, Andrade FH, Stahl JS. Functional and genomic changes in the mouse ocular motor system in response to light deprivation from birth. Journal of Neuroscience 2004; 24:161-9.

Ragge NK, Hartley C, Dearlove AM, Walker J, Russell-Eggitt I, Harris CM. Familial vestibulocerebellar disorder maps to chromosome 13q31q33: a new nystagmus locus. Journal of Medical Genetics 2003; 40: $37-41$.

Reinecke RD, Guo S, Goldstein HP. Waveform evolution in infantile nystagmus: an electro-oculo-graphic study of 35 cases. Binocular Vision 1988; 3:191-202.

Robinson DA, Gordon JL, Gordon SE. A model of smooth pursuit eye movements. Biological Cybernetics 1986; 55:43-57.

Swanzy HR. A Handbook of the Diseases of the Eye and Their Treatment. 5th ed, H.K.Lewis, London. 1895.

Tusa RJ, Mustari MJ, Burrows AF, Fuchs AF. Gaze-stabilizing deficits and latent nystagmus in monkeys with brief, early-onset visual deprivation: eye movement recordings. J Neurophysiol 2001; 86:651-61.

Virsu V, Rovamo J, Laurinen P, Näsänen R. Temporal contrast sensitivity and cortical magnification. Vision Research 1982; 22:12117. 